\title{
The finite basis problem for monoids of triangular boolean matrices
}

\author{
M. V. Volkov* \\ I. A. Goldberg \\ Department of Mathematics and Mechanics \\ Ural State University \\ 620083 Ekaterinburg, RUSSIA \\ Mikhail.Volkov@usu.ru, Goldberg@skbkontur.ru
}

\begin{abstract}
We show that the monoid of all $n \times n$ upper triangular boolean matrices has no finite identity basis whenever $n>3$. The identities of its submonoid consisting of matrices in which all diagonal entries are 1 possess a finite basis if and only if $n \leq 4$.
\end{abstract}

\section{The finite basis problem for matrix monoids}

An algebra $A$ is said to be finitely based if all identities holding in $A$ follow from a finite set of such identities (an identity basis of $A$ ); otherwise $A$ is called nonfinitely based. While every finite group is finitely based (Oates and Powell [5]), among finite monoids there exist nonfinitely based ones. The first example of a nonfinitely based finite monoid (due to Perkins [6]) was the Brandt monoid $B_{2}^{1}$ formed by the six matrices

$$
\left(\begin{array}{ll}
0 & 0 \\
0 & 0
\end{array}\right),\left(\begin{array}{ll}
1 & 0 \\
0 & 1
\end{array}\right),\left(\begin{array}{ll}
1 & 0 \\
0 & 0
\end{array}\right),\left(\begin{array}{ll}
0 & 1 \\
0 & 0
\end{array}\right),\left(\begin{array}{ll}
0 & 0 \\
1 & 0
\end{array}\right),\left(\begin{array}{ll}
0 & 0 \\
0 & 1
\end{array}\right)
$$

under the usual multiplication of $n \times n$-matrices:

$$
\left(\alpha_{i j}\right) \cdot\left(\beta_{i j}\right)=\left(\sum_{k=1}^{n} \alpha_{i k} \beta_{k j}\right) .
$$

*The first author's participation in the Workshop on Algebraic Systems, Formal Languages and Conventional and Unconventional Computation Theory was supported by a travel grant from the Russian Basic Research Foundation and by Kyoto Sangyo University. The first author is very much indebted to these organizations and to Professor Masami Ito for the invitation and his kind hospitality. 
We have not yet specified where 0 and 1 , the entries of the matrices in (1), come from and how they add and multiply. In fact, there is a certain degree of freedom here: since the above matrices have at most one non-zero entry in each row, the multiplication (2) works just the same provided that the symbols 0 and 1 obey the following rules:

$$
0 \cdot 0=0 \cdot 1=1 \cdot 0=0+0=0, \quad 1 \cdot 1=1+0=0+1=1,
$$

whereas the value of the sum $1+1$ does not matter at all. Therefore, one may conveniently think of $B_{2}^{1}$ as of a submonoid of the monoid $\mathscr{M}_{2}(F)$ of all $2 \times 2$-matrices over an arbitrary field $F$ and, in the same time, as of a submonoid of the monoid $\mathscr{B}_{2}$ of all binary relations over the 2-element set because the latter monoid can be identified with the monoid of all $2 \times 2$ matrices over the boolean semiring $\mathscr{B}=\langle\{0,1\} ;+, \cdot\rangle$ in which the operations satisfy the rules (3) and in addition $1+1=1$.

The twofold nature of $B_{2}^{1}$ is important in view of Sapir's powerful result [8] revealing that this monoid is inherently nonfinitely based. We recall that a finite monoid is said to be inherently nonfinitely based if it is contained in no locally finite finitely based variety. Since the variety generated by a finite monoid is locally finite, every inherently nonfinitely based finite monoid automatically is nonfinitely based; moreover, if the variety var $M$ generated by a finite monoid $M$ contains an inherently nonfinitely based monoid, then $M$ itself is inherently nonfinitely based and so $M$ is nonfinitely based. Therefore the fact that $B_{2}^{1}$ is inherently nonfinitely based immediately solves the finite basis problem for both the monoid $\mathscr{M}_{n}(F)$ of all $n \times n$-matrices over a finite field $F$ and the monoid $\mathscr{B}_{n}$ of all binary relations over the $n$-element set: for $n \geq 2$ these monoids are nonfinitely based. (Indeed, one only has to take into account that if $n \geq 2$ then $\mathscr{M}_{n}(F)$ and $\mathscr{B}_{n}$ contain submonoids isomorphic to $\mathscr{M}_{2}(F)$ and respectively $\mathscr{B}_{2}$, whence they both possess submonoids isomorphic to $B_{2}^{1}$-then Sapir's theorem applies.) An alternative proof of this result which does not depend on the notion of an inherently nonfinitely based monoid has been published in [12]; again, this proof is a byproduct of a more general approach.

Since a complete solution of the finite basis problem for full matrix monoids has been found, the next logical step in the general programme of classifying finite monoids with respect to the finite basis property may consist in investigating certain distinguished submonoids in $\mathscr{M}_{n}(F)$ and $\mathscr{B}_{n}$. Here the monoids of triangular matrices naturally come into the play. Let $\mathscr{T}_{n}(F)$ denote the submonoid of all upper triangular matrices in $\mathscr{M}_{n}(F)$ and let $\mathscr{T} \mathscr{B}_{n}$ be the monoid of all upper triangular boolean $n \times n$-matrices. 
In [14] we have studied and partially solved the finite basis problem for the monoids $\mathscr{T}_{n}(F)$ where $F$ is a finite field. We note that these monoids generate varieties which do not contain the monoid $B_{2}^{1}$ so Sapir's result does not apply here; neither does the approach from [12]. Nevertheless, we have proved that $\mathscr{T}_{n}(F)$ is nonfinitely based (and even inherently nonfinitely based) provided that $|F|>2$ and $n \geq 4$. The cases when the field $F$ has precisely 2 elements or when $|F|>2$ but $n=2,3$ still remain open; we can only prove that under these conditions the monoid $\mathscr{T}_{n}(F)$ is not inherently nonfinitely based but we do not know whether or not it is finitely based.

The first main result of the present note is to some extent similar to the cited result of [14]: we show that the monoid $\mathscr{T}_{\mathscr{B}}$ is nonfinitely based (and even inherently nonfinitely based) provided that $n \geq 4$. The reason behind this fact is that in the boolean situation the monoid $B_{2}^{1}$ does belong to the varieties generated by the monoids of triangular matrices of sufficiently big size. Again, in the cases when $n=2,3$ we only know that $\mathscr{T} \mathscr{B}_{n}$ is not inherently nonfinitely based but we do not know whether it is finitely based.

Our second main result solves the finite basis problem for a closely related family of submonoids in $\mathscr{B}_{n}$, namely, for the monoids $\mathscr{U} \mathscr{T} \mathscr{B}_{n}$ of all upper unitriangular boolean $n \times n$-matrices, that is, upper triangular boolean matrices in which all diagonal entries are 1 . We show that the monoids $\mathscr{U} \mathscr{T} \mathscr{B}_{n}$ are nonfinitely based whenever $n \geq 5$ and provide an explicit finite identity basis for each monoid $\mathscr{U} \mathscr{T} \mathscr{B}_{n}$ with $n=2,3,4$.

For the sake of completeness we mention that the monoids of all upper unitriangular $n \times n$-matrices over a finite field $F$ are finitely based. Indeed, these monoids are groups, and Oates-Powell's theorem [5] applies.

We summarize this mini-survey of the current state of art in studying the finite basis problem for finite monoids of matrices in the following table.

Table 1: The finite basis property of some finite monoids of $n \times n$-matrices

\begin{tabular}{|l|l|l|l|}
\hline \multirow{2}{*}{$\begin{array}{l}\text { The underlying } \\
\text { structure: }\end{array}$} & \multicolumn{3}{|c|}{ Type of matrices: } \\
\cline { 2 - 4 } $\begin{array}{l}\text { a finite } \\
\text { field } F\end{array}$ & $\begin{array}{l}\text { arbitrary } \\
\text { nonfinitely } \\
\text { based } \\
\text { for all } n \geq 2\end{array}$ & $\begin{array}{l}\text { nonfinitely based } \\
\text { if }|F|>2 \text { and } n \geq 4 ; \\
\text { unknown } \\
\text { if }|F|=2 \text { or } n=2,3\end{array}$ & $\begin{array}{l}\text { finitely based } \\
\text { for all } n \geq 2\end{array}$ \\
\hline $\begin{array}{l}\text { the boolean } \\
\text { semiring } \mathscr{B}\end{array}$ & $\begin{array}{l}\text { nonfinitely } \\
\text { based } \\
\text { for all } n \geq 2\end{array}$ & $\begin{array}{l}\text { nonfinitely based } \\
\text { if } n \geq 4 ; \\
\text { unknown if } n=2,3\end{array}$ & $\begin{array}{l}\text { nonfinitely based } \\
\text { if and only if } n \geq 5\end{array}$ \\
\hline
\end{tabular}


The reader who wants to place the results listed in Table 1 into a broader context can find a comprehensive report on the finite basis problem for finite monoids in [13].

\section{The identities of the monoid $\mathscr{T}_{B_{n}}$}

Theorem 2.1. The monoid $\mathscr{T}_{n}$ is inherently nonfinitely based if and only if $n \geq 4$.

Proof. First observe that for each $n \geq 4$ the monoid $\mathscr{T} \mathscr{B}_{n}$ contains a submonoid isomorphic to $\mathscr{T}_{\mathscr{B}_{4}}$. Therefore, as explained in Section 1 , it suffices to verify that the Brandt monoid $B_{2}^{1}$ belongs to the variety var $\mathscr{T}_{4}$. Consider the submonoid $B$ in $\mathscr{T} \mathscr{B}_{4}$ generated by the following two matrices:

$$
a=\left(\begin{array}{llll}
1 & 1 & 0 & 0 \\
0 & 0 & 0 & 1 \\
0 & 0 & 0 & 0 \\
0 & 0 & 0 & 1
\end{array}\right) \quad \text { and } \quad b=\left(\begin{array}{llll}
1 & 0 & 1 & 0 \\
0 & 0 & 0 & 0 \\
0 & 0 & 0 & 1 \\
0 & 0 & 0 & 1
\end{array}\right)
$$

Since for each matrix $\left(\gamma_{i j}\right) \in B$ one has $\gamma_{11}=\gamma_{44}=1$, the set $I$ of all matrices $\left(\delta_{i j}\right) \in B$ such that $\delta_{14}=1$ forms an ideal in $B$. A straightforward calculation shows that, besides $a, b$, and the identity matrix $e$, only the two matrices

$$
a b=\left(\begin{array}{cccc}
1 & 0 & 1 & 0 \\
0 & 0 & 0 & 1 \\
0 & 0 & 0 & 0 \\
0 & 0 & 0 & 1
\end{array}\right) \quad \text { and } \quad b a=\left(\begin{array}{cccc}
1 & 1 & 0 & 0 \\
0 & 0 & 0 & 0 \\
0 & 0 & 0 & 1 \\
0 & 0 & 0 & 1
\end{array}\right)
$$

belong to $B \backslash I$. This allows one to organize the following bijection between $B \backslash I$ and the set of non-zero matrices in $B_{2}^{1}$ :

$e \mapsto\left(\begin{array}{ll}1 & 0 \\ 0 & 1\end{array}\right), a b \mapsto\left(\begin{array}{ll}1 & 0 \\ 0 & 0\end{array}\right), a \mapsto\left(\begin{array}{ll}0 & 1 \\ 0 & 0\end{array}\right), b \mapsto\left(\begin{array}{ll}0 & 0 \\ 1 & 0\end{array}\right), b a \mapsto\left(\begin{array}{ll}0 & 0 \\ 0 & 1\end{array}\right)$,

and one easily checks that this bijection extends to an isomorphism between the Rees quotient $B / I$ and the monoid $B_{2}^{1}$. We see that $B_{2}^{1}$ turns out to be a morphic image of a submonoid in $\mathscr{T}_{\mathscr{B}_{4}}$ whence $B_{2}^{1}$ belongs to the variety generated by $\mathscr{T}_{\mathscr{B}}$.

It remains to verify that the monoids $\mathscr{T} \mathscr{B}_{2}$ and $\mathscr{T} \mathscr{B}_{3}$ are not inherently nonfinitely based. Since $\mathscr{T} \mathscr{B}_{2}$ embeds into $\mathscr{T} \mathscr{B}_{3}$, it suffices to consider the 
latter monoid. With a little help of a computer programme, we have checked that $\mathscr{T} \mathscr{B}{ }_{3}$ satisfies the identities

$$
x^{5}=x^{6} \quad \text { and } \quad(x y)^{5}(y x)^{5}(x y)^{5}=(x y)^{5} .
$$

Clearly, the first of these identities fails in any non-trivial group, and the second one can be shown to fail in the monoid $B_{2}^{1}$ : just substitute $\left(\begin{array}{ll}0 & 1 \\ 0 & 0\end{array}\right)$ for $x$ and $\left(\begin{array}{ll}0 & 0 \\ 1 & 0\end{array}\right)$ for $y$. Hence the variety $\operatorname{var} \mathscr{T}_{3}$ contains neither $B_{2}^{1}$ nor non-trivial groups. From [9, Theorem 2] we conclude that the monoid $\mathscr{T} \mathscr{B}_{3}$ is not inherently nonfinitely based.

Remark 2.1. The monoids $\mathscr{T}_{n}$ have been extensively studied by Pin and Straubing [7]. Using the techniques of formal language theory, they have proved that the pseudovariety generated by these monoids coincides with the well known pseudovariety PJ generated by power monoids of $\mathscr{J}$-trivial finite monoids. Since the Brandt monoid $B_{2}^{1}$ is known to belong to PJ (see, for instance, $\left[1\right.$, Section 11.6]), this result implies that $B_{2}^{1}$ belongs to the variety var $\mathscr{T} \mathscr{B}_{m}$ for some $m$, and thus, the monoids $\mathscr{T}_{n}$ with $n \geq m$ are inherently nonfinitely based. Our direct and rather simple approach gives the exact value of this $m$, namely, $m=4$.

\section{The identities of the monoid $\mathscr{U} \mathscr{T} \mathscr{B}_{n}$}

We obtain the main result of this section by identifying the collection of all identities of the monoid $\mathscr{U} \mathscr{T} \mathscr{B}_{n}$ with a set of identities previously studied by Blanchet-Sadri [2-4]. Let us first define the latter set. We fix a countably infinite alphabet $\Sigma=\left\{x, y, z, t, x_{1}, x_{2}, \ldots, y_{1}, y_{2}, \ldots\right\}$; as usual, $\Sigma^{*}$ stands for the free monoid over $\Sigma$. If $u, v \in \Sigma^{*}$ are two words, we say that $u$ is a scattered subword of $v$ whenever there exist words $u_{1}, \ldots, u_{n}, v_{0}, v_{1}, \ldots, v_{n-1}, v_{n} \in$ $\Sigma^{*}$ such that

$$
u=u_{1} \cdots u_{n} \quad \text { and } \quad v=v_{0} u_{1} v_{1} \cdots v_{n-1} u_{n} v_{n}
$$

in other terms, this means that one can extract $u$ treated as a sequence of letters from the sequence $v$. We denote by $J_{k}$ the set of all identities $u=v$ such that the words $u$ and $v$ have the same set of scattered subwords of length $\leq k$. Clearly, we have

$$
J_{1} \supseteq J_{2} \supseteq \cdots \supseteq J_{k} \supseteq \cdots
$$


Blanchet-Sadri [2-4] has established the following facts about the identity sets $J_{k}$ :

Proposition 3.1. a) The identities

$$
x^{2}=x, x y=y x
$$

form a basis of the set $J_{1}$.

b) The identities

$$
x y x z x=x y z x,(x y)^{2}=(y x)^{2}
$$

form a basis of the set $J_{2}$.

c) The identities

$$
\begin{gathered}
x y x^{2} z x=x y x z x, x y z x^{2} t z=x y x z x^{2} t z, z y x^{2} z t x=z y x^{2} z x t x \\
(x y)^{3}=(y x)^{3}
\end{gathered}
$$

form a basis of the set $J_{3}$.

d) The sets $J_{k}$ with $k \geq 4$ are not finitely based.

It should be stressed that speaking about identity bases we always mean bases in the sense of monoid identities. For instance, we have not included in the above base for $J_{2}$ the identity $x^{3}=x^{2}$ (which certainly belongs to $J_{2}$ ) because in the monoid setting it can be deduced from the identity $x y x z x=x y z x$ by substituting 1 for $y$ and $z$. However, as observed in [13, p.173], the property of a monoid to be finitely based or nonfinitely based does not depend on using the semigroup or the monoid deduction rules.

The following proposition relates the identity sets $J_{k}$ and the monoid $\mathscr{U} \mathscr{T} \mathscr{B}_{n}$ :

Proposition 3.2. For each positive integer $k$, the set $J_{k}$ coincides with the set $I_{k}$ of all identities holding in the monoid $\mathscr{U} \mathscr{T} \mathscr{B}_{k+1}$.

Proof. In order to prove the inclusion $I_{k} \subseteq J_{k}$ we use an argument due to Straubing [10]. Given a word $w=x_{1} \cdots x_{m}$ with $x_{1}, \ldots, x_{m} \in \Sigma$ and $m \leq k$, we define the morphism $\varphi_{w}: \Sigma^{*} \rightarrow \mathscr{U} \mathscr{T} \mathscr{B}_{k+1}$ by the rule

$$
\left(x \varphi_{w}\right)_{i j}= \begin{cases}1 & \text { if } i=j \\ 1 & \text { if } x=x_{i} \text { and } j=i+1 \\ 0 & \text { in the other cases. }\end{cases}
$$


For instance, if $w=x y x$ and $k=3$, then

$$
x \varphi_{w}=\left(\begin{array}{cccc}
1 & 1 & 0 & 0 \\
0 & 1 & 0 & 0 \\
0 & 0 & 1 & 1 \\
0 & 0 & 0 & 1
\end{array}\right), y \varphi_{w}=\left(\begin{array}{cccc}
1 & 0 & 0 & 0 \\
0 & 1 & 1 & 0 \\
0 & 0 & 1 & 0 \\
0 & 0 & 0 & 1
\end{array}\right), \quad \text { and } z \varphi_{w}=\left(\begin{array}{cccc}
1 & 0 & 0 & 0 \\
0 & 1 & 0 & 0 \\
0 & 0 & 1 & 0 \\
0 & 0 & 0 & 1
\end{array}\right)
$$

for all letters $z \neq x, y$.

Lemma 3.3. For each word $v \in \Sigma^{*}$, the word $w$ is a scattered subword of $v$ if and only if $\left(v \varphi_{w}\right)_{1 m+1}=1$.

Proof. Let $v=y_{1} \cdots y_{n}$ for some $y_{1}, \ldots, y_{n} \in \Sigma$, and let $y_{s} \varphi_{w}=\left(\alpha_{i j}^{(s)}\right)$. Then, using the multiplication rule (2) $n-1$ times, we arrive at the following expansion of the element $\left(v \varphi_{w}\right)_{1 m+1}$ :

$$
\left(v \varphi_{w}\right)_{1 m+1}=\sum_{1 \leq j_{1} \leq \cdots \leq j_{n-1} \leq m+1} \alpha_{1 j_{1}}^{(1)} \alpha_{j_{1} j_{2}}^{(2)} \cdots \alpha_{j_{n-1} m+1}^{(n)} .
$$

If $w=x_{1} \cdots x_{m}$ is a scattered subword of $v$, then there exists a sequence $1 \leq s_{1}<\cdots<s_{m} \leq n$ such that $x_{i}=y_{s_{i}}$ for every $i=1, \ldots, m$. Then all factors in the product

$$
\begin{aligned}
\alpha_{11}^{(1)} \cdots \alpha_{11}^{\left(s_{1}-1\right)} & \alpha_{12}^{\left(s_{1}\right)} \alpha_{22}^{\left(s_{1}+1\right)} \cdots \alpha_{22}^{\left(s_{2}-1\right)} \alpha_{23}^{\left(s_{2}\right)} \cdots \\
& \cdots \alpha_{m m}^{\left(s_{m-1}+1\right)} \cdots \alpha_{m m}^{\left(s_{m}-1\right)} \alpha_{m m+1}^{\left(s_{m}\right)} \alpha_{m+1 m+1}^{\left(s_{m}+1\right)} \cdots \alpha_{m+1 m+1}^{(n)}
\end{aligned}
$$

are equal to 1 , whence the product itself equals 1 and so does the sum in the right hand side of (8) of which this product is one of the summands. Thus, $\left(v \varphi_{w}\right)_{1 m+1}=1$.

Conversely, if $\left(v \varphi_{w}\right)_{1 m+1}=1$, then at least one of the summands in the right hand side of (8) is equal to 1 . Let

$$
\alpha_{1 j_{1}}^{(1)} \alpha_{j_{1} j_{2}}^{(2)} \cdots \alpha_{j_{n-1} m+1}^{(n)}=1 .
$$

By the definition of the mapping $\varphi_{w}$, we have $\alpha_{i j}^{(s)}=0$ whenever $i \neq j, j+1$. Therefore the increasing sequence $j_{0}=1 \leq j_{1} \leq \cdots \leq j_{n-1} \leq m+1=j_{n}$ contains exactly $m$ "jumps" of the form $j_{r}<j_{r+1}=j_{r}+1$. If we denote by $s_{i}, i=1, \ldots, m$, the position of the $i^{t h}$ such jump, then the product $\alpha_{1 j_{1}}^{(1)} \alpha_{j_{1} j_{2}}^{(2)} \cdots \alpha_{j_{n-1} m+1}^{(n)}$ can be written in the form (9), and since all the factors $\alpha_{i+1+1}^{\left(s_{i}\right)}$ are equal to 1 , we conclude from (7) that $y_{s_{i}}=x_{i}$ for every $i$. Thus, $w=x_{1} \cdots x_{m}$ is indeed a scattered subword of $v=y_{1} \cdots y_{n}$. 
Now in order to prove that $I_{k} \subseteq J_{k}$, take any identity $u=v$ holding in the monoid $\mathscr{U} \mathscr{T} \mathscr{B}_{k+1}$. Then $u \varphi=v \varphi$ under an arbitrary morphism $\varphi: \Sigma^{*} \rightarrow \mathscr{U} \mathscr{T} \mathscr{B}_{k+1}$. In particular, $u \varphi_{w}=v \varphi_{w}$ for every word $w \in \Sigma^{*}$ of length $m \leq k$, whence $\left(u \varphi_{w}\right)_{1 m+1}=\left(v \varphi_{w}\right)_{1 m+1}$. By Lemma 3.3, the latter equality means that $w$ is a scattered subword of $u$ if and only if it is a scattered subword of $v$. Therefore the identity $u=v$ belongs to set $J_{k}$.

The converse inclusion $J_{k} \subseteq I_{k}$ amounts to saying that for every identity $u=v$ in $J_{k}$ and for every morphism $\varphi: \Sigma^{*} \rightarrow \mathscr{U} \mathscr{T}_{\mathscr{B}_{k+1}}$ one has

$$
(u \varphi)_{\ell m}=(v \varphi)_{\ell m}
$$

for each $\ell \leq m$. By symmetry, it suffices to verify that $(u \varphi)_{\ell m}=1$ whenever $(v \varphi)_{\ell m}^{\prime}=1$. Thus, consider $v=y_{1} \cdots y_{n}$ for some $y_{1}, \ldots, y_{n} \in \Sigma$, and let $y_{s} \varphi=\left(\alpha_{i j}^{(s)}\right)$. Then

$$
(v \varphi)_{\ell m}=\sum_{\ell \leq j_{1} \leq \cdots \leq j_{n-1} \leq m} \alpha_{\ell j_{1}}^{(1)} \alpha_{j_{1} j_{2}}^{(2)} \cdots \alpha_{j_{n-1} m}^{(n)}
$$

and since the sum on the right hand side is equal to 1 , one of its summands is equal to 1 . Let the product $\alpha_{\ell j_{1}}^{(1)} \alpha_{j_{1} j_{2}}^{(2)} \cdots \alpha_{j_{n-1} m}^{(n)}$ be such a summand. The number $p$ of "jumps" $j_{r}<j_{r+1}$ in the increasing sequence $j_{0}=\ell \leq j_{1} \leq$ $\cdots \leq j_{n-1} \leq m=j_{n}$ does not exceed $m-\ell \leq(k+1)-1=k$. If we denote the position of the $i^{\text {th }}$ such jump by $s_{i}$ and its increment $j_{r+1}-j_{r}$ by $d_{i}$, $i=1, \ldots, p$, then the chosen product can be written as

$$
\begin{aligned}
\alpha_{\ell \ell}^{(1)} \cdots \alpha_{\ell \ell}^{\left(s_{1}-1\right)} \alpha_{\ell \ell+d_{1}}^{\left(s_{1}\right)} \alpha_{\ell+d_{1} \ell+d_{1}}^{\left(s_{1}+1\right)} \cdots \alpha_{\ell+d_{1} \ell+d_{1}}^{\left(s_{2}-1\right)} \alpha_{\ell+d_{1} \ell+d_{1}+d_{2}}^{\left(s_{2}\right)} \cdots \\
\cdots \alpha_{m-d_{p} m-d_{p}}^{\left(s_{p-1}+1\right)} \cdots \alpha_{m-d_{p} m-d_{p}}^{\left(s_{p-1}-1\right)} \alpha_{m-d_{p} m}^{\left(s_{p}\right)} \alpha_{m m}^{\left(s_{p}+1\right)} \cdots \alpha_{m m}^{(n)}
\end{aligned}
$$

whence we conclude that

$$
\alpha_{\ell \ell+d_{1}}^{\left(s_{1}\right)}=\alpha_{\ell+d_{1} \ell+d_{1}+d_{2}}^{\left(s_{2}\right)}=\cdots=\alpha_{m-d_{p} m}^{\left(s_{p}\right)}=1 .
$$

Consider the scattered subword $y_{s_{1}} y_{s_{2}} \cdots y_{s_{p}}$ of the word $v$. Since the length of this subword is $\leq k$ and since the identity $u=v$ belongs to $J_{k}$, the word $u$ also has $y_{s_{1}} y_{s_{2}} \cdots y_{s_{p}}$ as a scattered subword. Thus, we can write $u$ as

$$
u=u_{0} y_{s_{1}} u_{1} y_{s_{2}} u_{2} \cdots u_{p-1} y_{s_{p}} u_{p}
$$

for some suitable words $u_{0}, u_{1}, \ldots, u_{p} \in \Sigma^{*}$. Therefore the entry $(u \varphi)_{\ell m}$ of the matrix $u \varphi$ has in its expansion the summand

$$
\left(u_{0}\right)_{\ell \ell} \alpha_{\ell \ell+d_{1}}^{\left(s_{1}\right)}\left(u_{1}\right)_{\ell+d_{1} \ell+d_{1}} \alpha_{\ell+d_{1} \ell+d_{1}+d_{2}}^{\left(s_{2}\right)} \cdots\left(u_{p-1}\right)_{m-d_{p} m-d_{p}} \alpha_{m-d_{p} m}^{\left(s_{p}\right)}\left(u_{p}\right)_{m m}
$$


which is equal to 1 in view of (10) and of the fact that all diagonal entries of matrices in $\mathscr{U} \mathscr{T} \mathscr{B}_{k+1}$ are 1 . Hence $(u \varphi)_{\ell m}=1$ as required.

From Propositions 3.1 and 3.2, we immediately obtain

Theorem 3.4. The monoid $\mathscr{U} \mathscr{T} \mathscr{B}_{n}$ is nonfinitely based for all $n \geq 5$. The monoids $\mathscr{U} \mathscr{T} \mathscr{B}_{2}, \mathscr{U} \mathscr{T} \mathscr{B}_{3}$, and $\mathscr{U} \mathscr{T} \mathscr{B}_{4}$ are finitely based, and the identity systems (4), (5), and respectively (6) may serve as their identity bases.

Remark 3.1. It is fairly easy to see that for each $n$ the variety var $\mathscr{U} \mathscr{T} \mathscr{B}_{n}$. contains neither $B_{2}^{1}$ nor non-trivial groups whence none of the monoids $\mathscr{U} \mathscr{T} \mathscr{B}_{n}$ are inherently nonfinitely based by [9, Theorem 2].

Remark 3.2. The approach used in our proof of Theorem 3.4 can be also applied in order to provide a partial answer to Problem 6.3 in [13], namely, to solve the finite basis problem for the monoids $\mathscr{C}_{n}$ of order preserving and decreasing transformations of a chain with $n$ elements. (Recall that a transformation $\alpha$ of the chain $\langle X, \leq\rangle$ is called order preserving if $i \leq j$ implies $i . \alpha \leq j . \alpha$ for all $i, j \in X$ and decreasing if $i . \alpha \leq i$ for every $i \in X$.) It can be shown that the monoid $\mathscr{C}_{n}$ satisfies precisely the same identities as the monoid $\mathscr{U} \mathscr{T} \mathscr{B}_{n}$, and therefore, $\mathscr{C}_{n}$ is finitely based if and only if $n \leq 4$. The proof of this result will be published elsewhere.

Remark 3.3. Using Simon's celebrated characterization of piecewise testable languages, Straubing [10] has shown that the monoids $\mathscr{U} \mathscr{T} \mathscr{B}_{n}$ generate the pseudovariety $\mathbf{J}$ of all $\mathscr{J}$-trivial finite monoids. This nice result, however, does not seem to shed any extra light on the finite basis problem for the monoids $\mathscr{U} \mathscr{T} \mathscr{B}_{n}$ (compare with Remark 2.1).

Remark 3.4. Straubing and Thérien [11] have observed that the monoid $\mathscr{U} \mathscr{T} \mathscr{B}_{n}$ admits the following compatible partial order: $\left(\alpha_{i j}\right) \leq\left(\beta_{i j}\right)$ if for $i, j$ either $\alpha_{i j}=\beta_{i j}$ or $\alpha_{i j}=1$ while $\beta_{i j}=0$. On the other hand, from their elegant proof of Simon's theorem [11, p.397] one can extract the following

Proposition 3.5. Let $M$ be a monoid with a compatible partial order $\leq$ under which 1 is the greatest element. If $k+1$ is the length of the longest chain in $\langle M, \leq\rangle$, then $M$ satisfies every identity in the set $J_{k}$.

It is easy to calculate that the length of the longest chain in the monoid $\mathscr{U} \mathscr{T} \mathscr{B}_{n}$ with the above ordering is $\frac{n(n-1)}{2}+1$ so the application of Proposition 3.5 yields that $\mathscr{U} \mathscr{T} \mathscr{B}_{n}$ satisfies all identities in $J_{\frac{n(n-1)}{2}}$. Our Proposition 3.2 shows that in fact $\mathscr{U} \mathscr{T} \mathscr{B}_{n}$ satisfies all identities in $J_{n-1}$ and this is the best possible result. 


\section{References}

[1] J. Almeida, Finite Semigroups and Universal Algebra, World Scientific, Singapore, 1995.

[2] F. Blanchet-Sadri, Games, equations and the dot-depth hierarchy, Comput. Math. Appl. 18 (1989) 809-822.

[3] F. Blanchet-Sadri, Equations and dot-depth one, Semigroup Forum 47 (1993) 305-317.

[4] F. Blanchet-Sadri, Equations and monoid varieties of dot-depth one and two, Theor. Comp. Sci. 123 (1994) 239-258.

[5] S. Oates and M. B. Powell, Identical relations in finite groups, J. Algebra 1 (1964) 11-39.

[6] P. Perkins, Bases for equational theories of semigroups, J. Algebra 11 (1969) 298-314.

[7] J-E. Pin and H. Straubing, Monoids of upper triangular matrices, in G. Pollák, Št. Schwarz and O. Steinfeld (eds), Semigroups. Structure and Universal Algebraic Problems [Colloquia Mathematica Societatis János Bolyai 39], NorthHolland, Amsterdam-Oxford-New York, 1985, 259-272.

[8] M. V. Sapir, Problems of Burnside type and the finite basis property in varieties of semigroups, Izv. Akad. Nauk SSSR, Ser. Mat. 51 (1987) 319-340 [Russian; Engl. translation Math. USSR-Izv. 30 (1987) 295-314].

[9] M. V. Sapir, Inherently nonfinitely based finite semigroups, Mat. Sb. 133 (1987) no.2, 154-166 [Russian; Engl. translation Math. USSR-Sb. 61 (1988) 155-166].

[10] H. Straubing, On finite $\mathscr{J}$-trivial monoids, Semigroup Forum 19 (1980) 107110.

[11] H. Straubing and D. Thérien, Partially ordered finite monoids and a theorem of I. Simon, J. Algebra 119 (1988) 393-399.

[12] M. V. Volkov, On finite basedness of semigroup varieties, Mat. Zametki 45 (1989) no.3, 12-23 [Russian; Engl. translation Math. Notes 45 (1989) 187194].

[13] M. V. Volkov, The finite basis problem for finite semigroups, Sci. Math. Japon. 53 (2001) 171-199.

[14] M. V. Volkov and I. A. Goldberg, Identities of semigroups of triangular matrices over finite fields, Mat. Zametki 73 (2003) no.4, 502-510 [Russian; Engl. translation Math. Notes 73 (2003) no.4, 474-481]. 